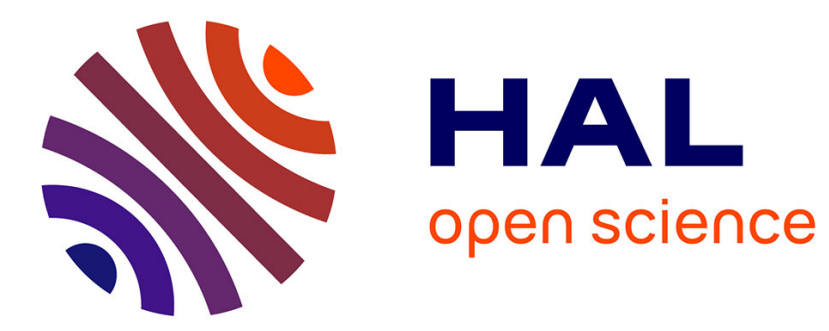

\title{
DU FRATRICIDE EN ALBANIE
}

Sophie Fierdepied

\section{To cite this version:}

Sophie Fierdepied. DU FRATRICIDE EN ALBANIE. Sud / Nord : folies et cultures, 2001, Marges, 15, pp.15-22. 10.3917/sn.015.0015 . halshs-01352752

\section{HAL Id: halshs-01352752 \\ https://shs.hal.science/halshs-01352752}

Submitted on 9 Aug 2016

HAL is a multi-disciplinary open access archive for the deposit and dissemination of scientific research documents, whether they are published or not. The documents may come from teaching and research institutions in France or abroad, or from public or private research centers.
L'archive ouverte pluridisciplinaire HAL, est destinée au dépôt et à la diffusion de documents scientifiques de niveau recherche, publiés ou non, émanant des établissements d'enseignement et de recherche français ou étrangers, des laboratoires publics ou privés. 


\section{DU FRATRICIDE EN ALBANIE}

Sophie Fierdepied-Biba

ERES $\mid \ll$ Sud/Nord »

$2001 / 2 \mathrm{n}^{0} 15$ | pages 15 à 22

ISSN 1265-2067

ISBN 2865868818

Article disponible en ligne à l'adresse :

http://www.cairn.info/revue-sud-nord-2001-2-page-15.htm

\section{Pour citer cet article :}

Sophie Fierdepied-Biba, « Du fratricide en Albanie », Sud/Nord 2001/2 (n 15), p. 15-22. DOI 10.3917/sn.015.0015

Distribution électronique Cairn.info pour ERES.

(C) ERES. Tous droits réservés pour tous pays.

La reproduction ou représentation de cet article, notamment par photocopie, n'est autorisée que dans les limites des conditions générales d'utilisation du site ou, le cas échéant, des conditions générales de la licence souscrite par votre établissement. Toute autre reproduction ou représentation, en tout ou partie, sous quelque forme et de quelque manière que ce soit, est interdite sauf accord préalable et écrit de l'éditeur, en dehors des cas prévus par la législation en vigueur en France. Il est précisé que son stockage dans une base de données est également interdit. 


\section{Sophie Fierdepied-Biba}

\section{Du fratricide en Albanie}

Entre l'enfer et le paradis, il y avait un mur, très haut et très difficile à franchir. Les Européens (ceux de l'Ouest, s'entend) décidèrent de monter les uns sur les autres pour se hisser jusqu'en haut, puis de tirer à eux les suivants pour les aider à monter. Aussi atteignirent-ils tous le paradis. Les Albanais, quant à eux, n'eurent de cesse de se bousculer, de s'écraser, s'accrochant à celui qui était parvenu à se hisser un peu, le faisant aussitôt retomber... aucun d'entre eux ne put échapper à l'enfer.

Cette histoire, racontée avec un humour cynique par les Albanais eux-mêmes, nous révèle à quel point ce peuple vit sa situation comme une fatalité d'être, tels qu'ils se décrivent : maudits, destinés à l'enfer quoi qu'ils fassent, héritiers d'une histoire pétrie de violence qui fait d'eux un peuple à part, à l'écart, en marge...

En effet, les Albanais disent avoir, depuis longtemps déjà, cette particularité, cette " malédiction ", celle d'être un peuple voué à la marginalité. Mais en marge de qui ou de quoi?

Historiquement tout d'abord, ce pays connut une dictature de plus de quarante ans si rigide et si excessive que, d'une part, il demeura à l'écart du monde capitaliste, mais que de plus, il rompit avec les autres pays du bloc communiste, provoquant ainsi son total isolement géographique, politique et culturel du reste du monde. Ce fut le régime le plus dur du bloc de l'Est. 
Marginalisé économiquement aussi, puisque l'Albanie reste encore aujourd'hui le pays le plus pauvre d'Europe. Marginalisé surtout par ces violences qui, de tout temps, restent le dénominateur commun de l'histoire des Albanais.

Pourtant, si les marginalités de ce petit pays du sud des Balkans sont incontestables, et font partie d'une réalité objective, il existe, chez chacun des membres de la communauté, un douloureux sentiment de marginalité, quant à lui beaucoup plus subjectif.

C'est plus particulièrement à ce douloureux sentiment de marginalité que nous nous intéresserons ici. Cette impression de marginalité trouverait-elle son origine dans les violences survenues au cours de l'histoire de ce pays? Des violences à ce point destructrices qu'elles auraient pu considérablement endommager les liens interindividuels et fraternels existants entre les membres de la communauté.

C'est dans l'après-coup d'un entretien clinique avec une personne réfugiée du Kosovo qu'un début d'élaboration du drame albanais nous fut possible. Le récit qui suit, et qui aurait pu se limiter à n'être qu'une simple étude de cas, se révéla par la suite être le point de départ de notre réflexion, tant il symbolise le drame d'une nation tout entière...

Un après-midi de février 2000, une partie de l'équipe de Handicap international, composée d'étudiantes en psychologie de l'université de Tirana ainsi que d'une interprète et de moi-même, se rend dans un des camps d'hébergement pour les réfugiés du Kosovo, ouvert au printemps 1999. Travaillant sur un programme de soutien psychologique, c'est dans une famille composée de cinq personnes que nous commençons nos visites.

Dan, l'un de ses membres, est un homme d'un peu plus de 40 ans, célibataire sans enfant, et qui vit depuis de longues années chez sa sœur et son beau-frère. Nous avons perçu chez lui des difficultés d'autonomie liées à un retard mental qui, bien que léger, ne lui permet pas de vivre seul. D'autre part, nous le savons enclin à la dépression mais, dans les camps où nous intervenons, ce trouble nous est presque devenu habituel tant il est fréquent.

Ce jour-là, il nous accueille avec la même marque d'affection que d'habitude, mais il est particulièrement sombre. Jamais encore Dan ne nous a raconté sa vie. Ce jour-là pourtant, il nous explique qu'il ne supporte plus que son histoire reste noyée au milieu des secrets de famille. Et c'est dans un accès de désespoir et de rage, dans un flot intarissable de paroles, qu'il va se confier à nous et nous confier une vie entière de misère et de souffrance.

Le père de Dan avait trois femmes (ce qui est encore tout à fait possible au Kosovo mais reste actuellement exceptionnel : la polygamie ne s'applique que dans certaines situations, comme les cas de stérilité chez la femme, pouvant pousser l'homme à prendre une concubine). Dan nous dira que sa mère, troisième et dernière 
concubine, malheureuse avec ses coépouses et régulièrement battue par son mari, décéda lorsquil était très jeune. À l'âge de 11 ans, suite à la mort de son père, il part vivre chez ses frères. Malheureusement, ils respectent peu, et maltraitent même ce petit frère qui souffre d'un retard mental, conséquence malheureuse d'une méningite qu'il contracta au cours de sa deuxième année de vie : «Ils me forçaient à garder les vaches et ne me donnaient que du pain... parfois même, j'ai dû manger de l'herbe et des racines tant j'avais faim..."

Nous comprenons qu'en plus de ce retard mental, Dan a toujours vécu dans un milieu qui n'a pas permis un développement psychoaffectif et psycho-intellectuel favorable. D'autre part, il n'a jamais été à l'école, absence d'éducation qui rajoute à ses difficultés.

À sa majorité, il part vivre auprès de sa sœur et de son beau-frère (avec qui il se trouve encore aujourd'hui), auprès de qui il trouve un entourage suffisamment bon. Il demeure chez eux quelques années, puis est marié par sa famille. La femme qu'il épouse, nettement plus âgée que lui et alcoolique, l'exploite autant qu'elle le peut. Dan passe quasiment tout son temps sur ses terres, auprès de ses bêtes, sans prendre grand soin de lui. Sa sœur nous racontera qu'elle dut lui retirer les lambeaux de chaussettes qu'il portait depuis deux ans sans jamais les avoir enlevées : il venait de passer ces deux années avec ses bêtes, dans les montagnes, chassé par sa femme qui l'avait contraint à ne plus redescendre...

Histoire terrible que celle de Dan, quil nous confie au comble de l'émotion. Pourtant, le pire reste à venir pour lui. Sa vie bascule ce jour de Bajram, fête marquant la fin du ramadan, où ses frères, sous prétexte de l'inviter à boire le thé, lui tendent un guet-apens : ils se ruent sur lui et le bastonnent à mort. Pour Dan, il est clair qu'ils ont tenté de le tuer pour hériter de ses terres fertiles.

Il passera trois semaines dans le coma et en ressortira transformé : «Personne ne me reconnaissait : je n'ai plus jamais été le même qu'avant, ni physiquement ni moralement. " Depuis ce terrible jour, Dan, que sa femme a quitté, est retourné vivre auprès de sa sœur et de son beau-frère.

Nous ressortons de cet entretien bouleversées. Flutura, l'interprète avec laquelle je travaille, se dira hantée par ce récit, au point d'en avoir mal dormi. Elle me sollicite dès le lendemain, m'expliquant avoir besoin que nous reparlions de cet entretien. Je lui propose de poser en premier lieu les choses sur le papier, ce qu' elle fait. Elle peut alors me dire que ce qui l'a le plus touchée, hantée, bouleversée, c'est l'histoire de ce fratricide. Comment peut-on tenter de tuer son propre frère ? Elle essaie alors de se raccrocher au fait qu'il s'agissait peut-être de ses demi-frères, chose plus supportable pour elle. Pour s'en assurer, elle repose la question à Genta qui lui confirme qu'il sagissait bien de ses propres frères, de même père et de même mère. Julie pourra dire qu'elle aussi est bouleversée par ce récit de vie, et que pour elle comme pour les autres 
le plus horrible à entendre fut l'histoire de ce fratricide. Je leur propose alors de rediscuter et de tenter d'analyser cette étude de cas ensemble, car je sens que se joue un je-ne-sais-quoi d'une histoire commune autour " du frère ", et que la terrible expérience de vie de Dan, Albanais du Kosovo, vient faire écho à une histoire collective, chez mes collègues albanaises d'Albanie.

Une voix off viendra illustrer mes réflexions tout au long de cet article. Cette voix off m'aide à comprendre ce pays, l'Albanie, et ce peuple; et je tiens à ce qu'elle résonne pour le lecteur aussi. C'est cette voix qui, depuis deux ans, me parle du désespoir des Albanais, et au travers de laquelle je perçois les mécanismes de défense mis en place face aux événements, à l'histoire insupportable de ce pays.

Cette voix cherche, elle aussi, désespérément, des explications, et ce par tous les moyens possibles : "C'est un complot politique ! C'est logique de la part des pays étrangers, de la part de l'extérieur... Il doit forcément y avoir des gens payés par les pays étrangers pour que ce soit autant l'anarchie ici... Les Grecs, les Italiens n'auraient aucun intérêt à ce que l'Albanie devienne forte économiquement, d'autant plus que nous sommes une main-d'œuvre rentable. Pour eux, nous sommes des chiens... non... nous ne sommes même pas des chiens ! Oui, c'est sûr, ce sont eux, les étrangers, qui viennent manipuler et payer les politiciens albanais pour que nous restions dans le chaos, pour que l'Albanie reste un enfer. Car ici, tu le vois bien, c'est l'enfer..."

Cette voix off vient à nouveau confirmer ce proverbe fataliste et tant de fois entendu : "Ce pays est béni mais son peuple est maudit. » Elle dit, malgré elle, les défenses psychiques qui vont permettre de donner une explication à l'insupportable, au non-représentable, et d'échapper à cette terrible réalité qui dirait : « Nous nous sommes détruits nous-mêmes."

Ce mécanismes de défense va consister à se tourner vers l'extérieur pour expliquer la cause de ses maux : «Dans ce pays, nous avons tout pour réussir : une terre fertile, des mines, du pétrole, un peuple fort... L'ennemi est extérieur. Ce sont eux, les étrangers, parce quils ont peur de notre force, qui manipulent, qui payent pour que nous nous autodétruisions... Les événements de 1997 : manipulation! La destruction du pays et de ses infrastructures en 1991, à l'ouverture : manipulation!»

Sans doute, ces paroles sont-elles héritées de la propagande divulguée par le dictateur Enver Hoxha, pour soumettre son peuple : l'ennemi est étranger et il veut nous détruire. Ainsi isola-t-il son pays et son peuple du reste du monde, sous prétexte que le monde entier voulait leur mort. Cette ignorance entretenue du monde extérieur laissait la place à tous les abus, à toutes les violences et à toutes les propagandes dont peut user un état autoritariste. Et à chaque changement d'orientation politique, c'était une nouvelle période de purge. 
Le dictateur, invoqué sous le nom d'« oncle Enver " (oncle paternel dans la traduction précise de l'albanais), avait donc, tel le " grand mâle de la horde sauvage » du mythe rapporté par Freud, le droit de vie ou de mort sur les siens. Il fit éclater le clan qui, de tout temps, fut la base de la solidarité et de la résistance albanaise, en modifiant la place de la femme dans la famille, en s'emparant de l'autorité ancestrale du chef de clan, et en organisant l'espionnage au sein même des familles. Il devint ainsi l'homme tout-puissant du pays, le père tyrannique de ce clan national quil enferma à l'intérieur de ses frontières et isola du reste du monde.

Mais au-delà de cet isolement géopolitique, ce fut en définitive une véritable mise en marge du monde du symbolique, de la civilisation que provoqua la dictature, et ce par cette initiative de destruction totale de l'ancien ordre social, des liens familiaux, des règles ancestrales, du code d'honneur, c'est-à-dire de tout ce qui faisait partie du monde symbolique et culturel de la communauté.

Peut-être toutes les dictatures doivent-elles nous ramener à cette sorte de préhistoire de la civilisation, et au mythe qui explique qu'en ce temps-là, un seul homme, le "grand mâle " tyrannique, possédait toutes les femmes et tous les biens de la tribu.

C'est sur ce mythe que s'appuie Freud dans Totem et tabou, pour nous expliquer la naissance de la civilisation. Après le meurtre du père tyrannique et tout-puissant, ses frères se livrèrent à une lutte acharnée pour savoir lequel d'entre eux lui succéderait. Cette lutte, cette tentative de fratricide resta sans vainqueur, abandonnée par la survenue de la culpabilité du parricide. C'est grâce à cette culpabilité, issue de l'ambivalence face à la figure du père, que les frères décident de se baser sur deux grandes lois : l'interdit du meurtre et l'interdit de l'inceste. Ces deux lois devaient alors réglementer leurs vies, les protéger les uns des autres, leur permettre de vivre les uns à côtélavec les autres. Ainsi naquit la civilisation : de la culpabilité qui conduit à la nécessité de dominer la violence.

Ce serait en quelque sorte, comme le dit encore Freud dans Malaise dans la civilisation, la victoire de la pulsion de vie sur la pulsion de mort, par refoulement de la seconde.

Et c'est bien au mythe de la " horde des frères " que je me surprends à penser lorsque je tente de comprendre l'histoire de l'Albanie de ces dix dernières années. Le parallèle peut, en effet, aisément s'entendre dans la chute de la dictature, véritable mise à mort symbolique du père tyrannique, et la destruction systématique de ce qui représente son pouvoir. Ce parricide symbolique que fut la révolution fut suivi d'une période de troubles et de luttes politiques qui générèrent les violents événements de 1997 puis de 1998.

Les événements de mars 1997, provoqués par la chute des systèmes pyramidaux, entraînèrent la révolte du peuple et la prise d'assaut des arsenaux. L'Albanie fut alors 
pendant quelques jours à feu et à sang. Ces émeutes se transformèrent bientôt en un véritable fratricide : plus de quatre mille morts sont dénombrés au cours de ces journées. Des bandes masquées se livrèrent au pillage, au vol. Les diverses infrastructures furent détruites, et avec elles l'économie, certes modeste, du pays (cette fois-ci, même les administrations, les musées et les écoles furent saccagés et pillés, alors qu’en 1991 n'avaient été touchés que les symboles de la dictature). Finalement, tout ce qui restait encore d'un semblant d'organisation et de fonctionnement fut anéanti, laissant place à l'anarchie, la loi du plus fort, la corruption...

Dès lors, la peur de l'autre fit son retour : «Le danger, c'est celui avec qui je bois le café, celui avec qui je travaille. Celui qui, hier, me tirait dessus, le visage protégé derrière sa cagoule, c'est peut-être tout simplement celui-là avec qui je plaisante en ce moment... "Amitié", "confiance", que veulent dire ces mots-là ? Quand je regarde mes compatriotes, j'ai l'impression de voir des ennemis partout..."

C'est la violence fratricide qui est symptomatique dans cette communauté. Et c'est parce que ce peuple fut projetée hors les lois humaines, hors la civilisation, en marge de celles-ci, que ce symptôme, signe de la réémergence de la pulsion de mort, est apparu.

En effet, la violence est toujours une expression pathologique de l'agressivité, donnée normale chez chaque individu. La pulsion agressive peut aussi bien appartenir à la pulsion de vie qu'à la pulsion de mort. Quand elle appartient à la pulsion de vie, elle répond à l'instinct d'autoconservation; quand elle appartient à la pulsion de mort, elle est de l'ordre de l'instinct destructif. Quand la pulsion de mort réémerge, les grandes lois humaines perdent tout leur sens, et plus rien ne l'empêche de réaliser et de dépasser les interdits.

Nous pourrions distinguer deux types de violence : l'une, conservatrice, répondant à Éros, l'autre, destructrice, répondant à la pulsion de mort, Thanatos. Dans le cas d'un fratricide, il s'agit très clairement d'un symptôme pulsionnel destructif. C'est un même que moi que je suis conduit à tuer, c'est-à-dire avec qui je partage une origine et une culture commune.

Ce qu'il y a de surprenant et de terrifiant, c'est la dimension collective de l'émergence de ce symptôme. Mais c'est, à n'en pas douter, dans l'histoire de ce pays, dans la violence qui fut faite à ce peuple, au cours de ces décennies de dictature, quil faut aller chercher les explications au syndrome d'autodestruction auquel nous assistons depuis dix ans en Albanie.

L'ombre du grand frère se dessine enfin, terrifiante, car les liens d'affection et de sang qui nous unissaient à lui ont été détruits, ont perdu leur sens, et le rendent étrangement inquiétant. Il est alors insupportable à la conscience de se confronter aux événements de ces dernières années, qui montrent un peuple en train de détruire son pays, qui décrivent un Albanais tuant un autre Albanais, qui parlent d'un peuple 
entier qui se méprise, et qui traite au quotidien ses compatriotes sans le moindre respect.

Aussi, quand Dan vient nous raconter son histoire, bouleversante, je n'en reste pas indemne, mais je m'interroge sur le fait qu'elle vienne résonner si fortement chez Flutura, Julie et Genta : "J'ai mal dormi ce soir-là... » L'ombre du fratricide hante la psyché de chacune, et provoque un malaise indicible, inconscient, insupportable. Et c'est bien dans ce traumatisme-là, celui de la rupture des liens symboliques, qu'il faut aller chercher pour comprendre ce qui vient faire effraction dans la psyché de mes collègues albanaises...

" [...] Comment veux-tu que nous ne nous regardions pas comme des loups, comment veux-tu que nous ne nous méfiions pas les uns des autres quand celui dont je dois me protéger est celui dont je devrais attendre du secours ? Certains d'entre nous en sont même arrivés à vendre nos sœurs pour alimenter les trottoirs de l'Ouest! C'est le sens humain que nous avons perdu, c'est le respect minimum pour nousmêmes qui nous manque."

Face à ce que la purification ethnique est venue anéantir, ou tenté d'anéantir, dans le psychisme des Kosovars, en relation avec les liens transgénérationnels et intercommunautaires, les Albanais avec lesquels nous avons travaillé ont souvent évoqué la résonance que cela provoquait avec leur propre histoire, leurs propres souffrances. Et il est vrai que les violences vécues par les Kosovars sont proches de celles vécues par les Albanais. Ce qui fait la différence entre les Albanais du Kosovo et les Albanais d'Albanie, c'est que pour ces seconds, ce ne sont pas ceux d'une ethnie différente qui sont venus attaquer et tenter d'anéantir ces liens. Pour ces seconds, c'est au sein même de la communauté que cela s'est passé : et preuve en est qu’à l'arrivée au pouvoir d'Enver Hoxha, un Albanais sur dix est mort sous la torture ou dans les prisons du pays. Aussi est-ce d'une épuration intra-communautaire dont il faudrait parler pour évoquer la dictature albanaise, faisant ainsi résonner l'importance de la rupture des liens à l'intérieur de cette communauté.

Ce qui provoque le traumatisme aujourd'hui, ce sont les violences de 1997 qui viennent faire écho à cette épuration intracommunautaire : ces récents événements fratricides lui ressemblent étrangement, car tout au long de ces presque cinquante années de dictature, il s'agissait avant tout, pour survivre, de se méfier de ses proches dont venaient le plus souvent la dénonciation, la trahison...

La violence paroxystique de 1997 ne serait donc que la conséquence d'une violence d'État qui ricoche, d'une violence fratricide qui se répète, qui vient parler de la rupture des liens, qui vient parler de la fragilité des lois fondamentales de l'humanité : "Tu ne tueras ni ton père ni ton frère. "

Et c'est là le traumatisme indicible, insupportable de l'Albanie, et je sens à quel point ce trou noir du traumatisme, que l'homme de la rue appelle plus aisément 
"malédiction », cette effraction dans la psyché est présente, sidérante : "Nous aurions tous besoin d'un thérapeute."

Et c'est bien de malédiction dont on parle lorsque les événements malheureux se reproduisent toujours et encore. Comment expliquer cette terrible répétition, cette suite constante de violences? Les Albanais sont-ils aussi maudits qu'ils ont l'habitude de le dire?

Nous pourrions remonter aussi loin que possible dans l'histoire du peuple albanais, que nous y trouverions, à chaque page, d'autres violences : invasions, conversions forcées, découpage aléatoire des frontières, entraînant la séparation du pays d'avec la moitié de sa population...

La psychanalyse nous a enseigné que toute histoire, tant qu'elle n'est pas élaborée, finit toujours par se répéter. Cette règle fonctionne aussi dans le collectif, et c'est pour cette raison que le devoir de mémoire est fondamental.

L'histoire récente de l'Albanie, c'est encore et toujours la répétition du mythe d'Abel et Caïn, et de leur sordide histoire : c'est par un fratricide que se conclut l'histoire des deux premiers frères du monde. Malgré tout, dans l'histoire de ces deux-là, il y avait un bon et un mauvais, un blanc et un noir. En Albanie, personne n'est blanc, personne ne se sent blanc, de manière inconsciente du moins. La culpabilité est là : celle, en premier lieu, d'avoir vécu dans ce système dictatorial, car, pour qu'une dictature s'installe, pour qu'elle perdure, il faut bien qu'une partie de la population en soit complice, il faut bien qu'une majorité regarde et laisse faire. En second lieu, il existe une culpabilité de s'être soumis à la dictature, d'avoir participé à la dénonciation d'autres, parfois pour se sauver soi-même. Mais aussi et enfin, une culpabilité de s'autodétruire aujourd'hui, de s'entretuer...

Dans le même temps, les Albanais voudraient bien que le monde cesse de les regarder comme des êtres étranges, dangereux, agressifs, peut-être pas déshumanisés, mais à coup sûr marginalisés, et les acceptent enfin. Mais le monde entier les regardet-il vraiment de cette manière-là ? Ou bien est-ce le regard quills se portent à euxmêmes, est ce, par culpabilité, ce que leur renvoie l'œil du monde... «L'œil était dans la tombe et regardait Caïn. "

L'histoire de l'Albanie, c'est le mythe de la " horde des frères ", mis en scène, rejeté du monde symbolique dans le monde réel. Reste à savoir si la culpabilité qui en émerge permettra de recréer du symbolisme, de renouer des liens fraternels, de faire enfin une place à la pulsion de vie et au retour des lois fondamentales. L'histoire récente de l'Albanie devra-t-elle se conclure comme le mythe de Caïn par sa damnation définitive ou bien laisser l'espoir que les frères trouveront de nouveaux codes, de nouvelles règles pour vivre de nouveau ensemble, sortir de la violence et de l'autodestruction qui maintiennent tout un peuple, tout un pays, dans la marginalité du monde symbolique? 